Published in: Journal of Applied Physics https://aip.scitation.org/journal/jap

Volume: 126 Issue: 15 Article Number: 155302 DOI: 10.1063/1.5114671 Published: OCT 21 2019

For personal use only

\title{
Influence of interfacial structural disorder and/or chemical interdiffusion on thermal boundary conductance for Ti/Si and Au/Si couples
}

\author{
Maïté Blank and Ludger Weber \\ Laboratoire de Métallurgie Mécanique, Ecole Polytechnique Fédérale de Lausanne, \\ Lausanne, Switzerland
}

\begin{abstract}
This work aims at experimentally assessing the effect of structural disorder and/or chemical interdiffusion on Thermal Boundary Conductance (TBC) at metal/dielectric interfaces. To this end the TBC of $\mathrm{Au} / \mathrm{Si}$ and Ti/Si interfaces was measured by Time Domain Thermoreflectance (TDTR) in the as-deposited state and after various heattreatments. Transmission Electron Microscopy (TEM) was used to determine the structure of the interface for each heat-treatment condition. The TBC at the Au/Si interface was found to be highest in the as-deposited state and to be somewhat reduced after heat-treatments. TEM images showed that the highest TBC was obtained when the interface contained a disordered transition layer with some degree of interdiffusion as present in the as-deposited state. Heat-treatments led to separation of species and to more abrupt interfaces with lower TBC. Whether this change in TBC was linked to disorder or to interdiffusion could not be distinguished. The TBC at the Ti/Si interfaces was observed to decrease with heat-treatment duration. TEM investigations revealed the presence of a thin disordered layer that did not evolve much with heat-treatments. The evolution of the TBC was hence rationalized by interdiffusion facilitated by heattreatments that most likely modified the diffusion zone thickness and chemical composition, as well as the disordered layer's chemical composition. These changes result in modified interfacial properties, which ultimately affect the TBC. A quantitative exploration of the TBC dependence on the composition was not possible in this work, due to the extreme thinness of the regions under consideration preventing any reliable STEM-EDX measurement.
\end{abstract}

\section{INTRODUCTION}

In the last decades, advances in many electronic devices have been critically dependent on the efficiency of heat removal on a local level. Compared to macroscopic solid-state heat conduction problems there are two complicating effects at the micro level: i) the thermal resistance at the interface may no longer be significantly smaller than the thermal resistance of the unperturbed bulk between interfaces, and ii) for characteristic 
length scales on the order of the mean free path of the main heat carriers the heat transfer becomes in part ballistic. While the first effect is ubiquitous in current electronic devices, the second is only seen in very small devices or at low temperature. The thermal boundary resistance or its inverse, the thermal boundary conductance can be modified in a certain range by designing the interface structure properly, i.e. typical values for metal/dielectric values range from 10 (Bi/H:diamond) - 800 (TiO/MgO) $\mathrm{MW} / \mathrm{m}^{2} \mathrm{~K}(1)$. The ballistic transport on the other hand sets a hard upper limit to thermal conductivity. Conceptually, interfaces are seen as a plane separating two different phases. In reality, however, they may not be a well-defined plane, but rather exhibit a finite roughness, contain adsorbed species, or form transition regions where species from either side are present in the opposite phase, or the combination of species from both side lead to an interphase that is crystallographically distinct from the two bounding phases. In this work, the thermal boundary conductance at metal/dielectric interfaces with some degree of intermixing and/or atomic disorder is investigated experimentally.

Extensive theoretical work has been carried out by several groups in an attempt to highlight possible effects of interdiffusion and structural disorder on the TBC (2-9). All these studies focused on dielectric/dielectric interfaces. These results should, however, be applicable to metal/dielectric interfaces since phonons are the heat carrying species across the interface in both cases.

A possible effect of the interdiffusion on the TBC between two FCC lattices was evaluated by Twu and Ho (2) using non-equilibrium molecular dynamic simulation (NEMD), while Beechem et al. (3) proposed a virtual crystal (VC) approach applied to the diffuse mismatch model (DMM). Both groups concluded that intermixing reduces the TBC. These results were rationalized by taking into account that interdiffusion creates additional phonon scattering, which impedes heat transport at the interface. NEMD simulations carried out by Stevens et al. (4) and MD simulations performed by Liang and Sun (5), however, showed an opposite trend. In both cases, intermixing was predicted to improve the TBC, which was attributed to the creation of vibrational states that bridge the materials properties on either side of the interface.

The role of interfacial structural disorder was explored by Beechem et al. (6) using a modification of the DMM. Giri et al. $(7,8)$ performed NEMD simulations at $\mathrm{Si} / \mathrm{Ge}, \mathrm{Si} / \mathrm{Ge} / \mathrm{Si}$ and $\mathrm{Ge} / \mathrm{Si} / \mathrm{Ge}$ interfaces, while Li et al. (9), studied the graphene/SiC system. All these contributions reached the conclusion that using interfaces made of amorphous materials is an efficient way to improve the TBC. At first sight, one might think that an interfacial region that is amorphous should rather lower the TBC because structural disorder promotes the existence of phonons with short mean free paths that are unlikely to be efficient for heat transport through the interface. However, the authors rationalized their findings by taking into account that i) disorder is likely to reduce the abruptness of the interfacial changes in materials properties on either side of the interface and ii) the amorphous phonon DOS is usually smoother than the crystalline DOS, allowing for a larger overlap between phonon modes on either side of the interface. Gordiz and Henry 
(10) used NEMD simulations to study $\mathrm{Si} / \mathrm{Ge}$ interfaces and reached slightly different results. Their model predicted the TBC to be roughly the same at the c-Si/c-Ge, c-Si/a$\mathrm{Ge}, \mathrm{a}-\mathrm{Si} / \mathrm{c}-\mathrm{Ge}$ and a-Si/a-Ge, although strong variations in DOS overlap were observed. Based on these calculations, the authors suggest that phonon scattering might not be the right mechanism to describe heat flow through interfaces, especially when amorphous materials are involved. They suggested that a picture involving correlation/coupling between modes should be used instead.

In view of these conflicting results depending on the modelling approach, experimental evidence for the effect on TBC of order and/or intermixing at interfaces would be of great help. Unfortunately, up to now, only very few authors focused on this question. Hopkins et al. (11) measured TBC at $\mathrm{Cr} / \mathrm{Si}$ samples, which underwent different surface treatments creating various conditions of interdiffusion and roughness. A general trend was identified, in which the TBC decreases when the interfacial region becomes larger and the silicon surface rougher. A clear differentiation between both effects, i.e. intermixing and roughening, was however not developed. More specifically, roughness was assumed to change but no quantitative measurements were given.

In the present contribution, we propose to study the evolution of the TBC at Ti/Si and $\mathrm{Au} / \mathrm{Si}$ interfaces, which underwent different heat-treatments. Ti/Si interfaces were observed to naturally form amorphous interlayers, located at the interface between both phases, when sputtered on H-terminated Si substrates $(12,13)$. Heat treatments at temperatures ranging from $350-450^{\circ} \mathrm{C}$ were further reported to allow for changes in the properties of this disordered region. Similarly, the formation of a highly disordered interfacial region at $\mathrm{Au} / \mathrm{RF}$-etched $\mathrm{Si}$ interfaces was recently reported by our group (14). Within this work, we will show that heat-treating those samples at $150^{\circ} \mathrm{C}, 245^{\circ} \mathrm{C}$ and $365^{\circ} \mathrm{C}$ for 15 minutes allows for a significant change of the interface structure and measured TBC. A study of the TBC evolution for these different systems as a function of the heat-treatment performed coupled with a detailed characterization of the samples that aims at providing information about i) possible interdiffusion between the materials on either side of the interface and ii) possible change in structural order in the interfacial region is believed to be a useful benchmark in studying the mechanisms involved when some amount of interdiffusion and/or structural disorder are present at the interface, while avoiding any change in interfacial bond strength and/or roughness due to a change of substrate preparation technique.

\section{EXPERIMENTAL METHOD}

\section{a. Sample preparation and characterisation}

All samples were prepared using $525 \mu$ m-thick [100] silicon wafers, which were RCA cleaned. Right before deposition, the native oxide layer was removed by a 3 minutes dip into a $1 \% \mathrm{HF}$ aqueous solution, resulting in a passivated $\mathrm{H}$-terminated surface (15).

$\mathrm{Au} / \mathrm{Si}$ couples were produced in an Alliance-Concept DP 650 sputtering machine. Each wafer underwent a 60s argon bombardment with a power of $100 \mathrm{~W}$, before being 
covered by approximately $80 \mathrm{~nm}$ of gold. One sample was kept in this so-called "asdeposited" state, while the others were heat-treated using a custom built oven operating at $150^{\circ} \mathrm{C}, 245^{\circ} \mathrm{C}$ and $365^{\circ} \mathrm{C}$ under a vacuum level $<510^{-6} \mathrm{mbar}$. The heat-treatment time was set to $15 \mathrm{~min}$ (the exact temperature cycles can be found in the Supplemental Material, Fig. S1). A $20 \mathrm{~nm}$ thick Cr cap layer was then added on top of each sample to act as a transducer.

$\mathrm{Ti} / \mathrm{Si}$ couples were produced using a Pfeiffer SPIDER 600 sputtering machine. Each sample was first covered by a thin Ti layer. One of them was kept in this so-called "asdeposited" state, while the others were in-situ heat-treated at $350^{\circ} \mathrm{C}$ during 30 minutes, $2 \mathrm{~h}$, and $3 \mathrm{~h}$, respectively, under a vacuum of $<5 \cdot 10^{-6}$ mbar. All samples were finally capped with an aluminium layer meant to serve as a transducer and a protection against oxidation. The titanium layer thickness was set to approximately $6 \mathrm{~nm}$. On the one hand, the use of such a thin layer improves the TDTR measurement sensitivity to the TBC, which might typically be reduced for larger layers because of both a low thermal conductivity of titanium and a large thermal boundary conductance at the Ti/Si interface. On the other hand, knowing that the electron-phonon coupling parameter of titanium is $>10^{17} \mathrm{~W} / \mathrm{m}^{3} \mathrm{~K}(16)$, a $6 \mathrm{~nm}$-thick layer is thick enough to ensure that the temperature difference between the electrons and the phonons has already reached a minimum. This ensures that the TBC is independent of any size effect (17). The Al cap layer was set to approximately $65 \mathrm{~nm}$.

X-Ray reflectivity was performed with a Panalytical Empyrean diffractometer to measure $\mathrm{Cr}, \mathrm{Au}, \mathrm{Al}$ and $\mathrm{Ti}$ layers thicknesses in $\mathrm{Au} / \mathrm{Si}$ and $\mathrm{Ti} / \mathrm{Si}$ samples respectively. The GenX software (18) was used to fit the obtained curves. The fitting parameters were the metallic layer thickness, roughness and density, as well as the substrate roughness and density. The presence of a disordered intermixed interfacial region at the metal/Si interface was taken into account assuming a 50:50 at-pct composition ratio. Such an approximation was already shown to improve the overall fit quality and thus provide precise measurement for the thickness of all layers made of pure metal (14). The thickness of each layer for each sample can be found in Table S.1. of the Supplemental Material.

As for the intermixed disordered interfacial regions, their detailed structure and thickness was determined by TEM using either a Tecnai Osiris microscope operating at $200 \mathrm{keV}$ or a FEI Titan Themis Cs-corrected microscope operating at $200 \mathrm{keV}$. All samples were mechanically polished until electron transparency was achieved. For $\mathrm{Au} / \mathrm{Si}$ samples, ion milling was further performed at $-100^{\circ} \mathrm{C}$ and $0.2 \mathrm{keV}$. None of the $\mathrm{Ti} / \mathrm{Si}$ samples were ion milled since the process was observed to reduce sample quality because of redeposition.

\section{b. Thermal boundary conductance measurement}

The thermal boundary conductance of each sample was measured using Time Domain Thermoreflectance. Details of the setup employed have already been given elsewhere $(19,20)$. In a nutshell, it consists of a pump-probe technique, in which the pump heats 
the sample surface periodically. This creates variations in the sample reflectivity, which are monitored by the probe. The technical specifications of the setup used within this work are as follows: the laser source was a COHERENT Mira 900 operating at a wavelength of $785 \mathrm{~nm}$ with a repetition rate of $76 \mathrm{MHz}$. The pump was modulated at a frequency of $10.7 \mathrm{MHz}$ using an Electro-Optic Modulator (EOM, Model 350-160, ConOptics Inc.). Full cooling curves were recorded for delay times ranging from 0 to $4 \mathrm{~ns}$ using a delay stage (M-531-PD1, Physik Instrument GmbH \& Co.) mounted on the pump optical path. The target fluence was set to $0.2 \mathrm{~mJ} / \mathrm{cm}^{2}$. The exact values were obtained for each experiment by measuring the beam spot size and power using a CMOS camera and a power meter (Tuner, Gentec EO Inc.) respectively. A spot size with a $1 / \mathrm{e}^{2}$ radius close to $4 \mu \mathrm{m}$ and beam power of roughly $7 \mathrm{~mW}$ were used for all experiments. The signal obtained was collected using a fast photodiode (Thorlabs, DET 10A), before being frequency filtered, pre-amplified and fed into a ZI-HF2 lock-in amplifier (Zurich Instruments, Zurich, Switzerland). The signal-to-noise ratio was improved by using the so-called "two-tint" technique, which consists in differentiating the pump and probe beam in terms of polarization and wavelength.

The scheme proposed by Cahill (21) was used to extract TBC values from the X/Y curves (i.e. the ratio of the in-phase to out-of-phase amplitude with regard to the modulation frequency of the EOM). Both the TBC and the substrate thermal conductivity were used as fitting parameters. The $\mathrm{Al}, \mathrm{Ti}, \mathrm{Cr}, \mathrm{Au}$ layer thicknesses were taken from XRR measurements, while the thicknesses of the intermixed disordered interfacial region (present in as-deposited $\mathrm{Au} / \mathrm{Si}$ and for all heat treatments in $\mathrm{Ti} / \mathrm{Si}$ ) were measured on TEM images. The $\mathrm{Al}, \mathrm{Ti}, \mathrm{Cr}$ and $\mathrm{Au}$ thermal conductivities and heat capacities were taken from the literature (22). The thermal conductivity and the heat capacity of the intermixed disordered interfacial region were evaluated following the same procedure as described in (14) and detailed in section IV.a.. Fig. 1 shows some examples of raw data and corresponding fits obtained for the Ti/Si couples. A good signal to noise ratio, as well as a satisfactory fit quality was obtained in all cases.

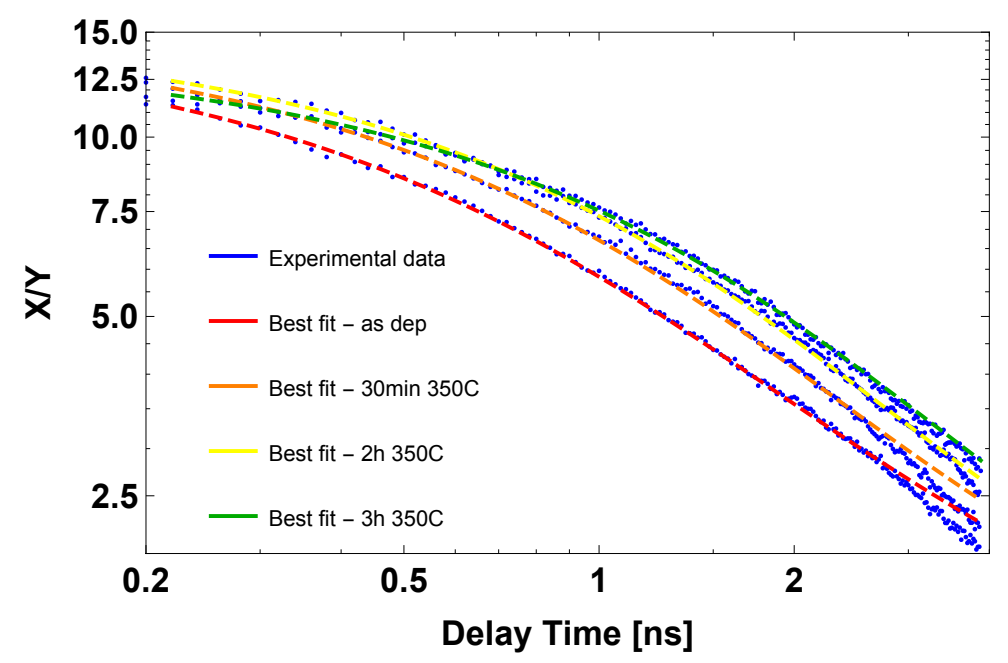

Fig. 1: TDTR raw signal and fitting curve obtained for Ti/Si samples in the as-deposited state and after heat-treatments of $15 \mathrm{~min}, 2 \mathrm{~h}$ and $3 \mathrm{~h}$. 


\section{RESULTS}

\section{a. Detailed structure of the interface}

\section{i. Au/Si interface}

Fig. 2 shows HRTEM images of the $\mathrm{Au} / \mathrm{Si}$ couple in the as-deposited state and after 15 minutes heat treatments at $150^{\circ} \mathrm{C}, 245^{\circ} \mathrm{C}$ and $365^{\circ} \mathrm{C}$. In all cases, the crystalline silicon, located on the left-hand side of the images is neighbouring an interfacial region, described hereafter for each sample, followed by crystalline gold. In the as-deposited sample, the interfacial region is $3.7 \mathrm{~nm}$ thick and exhibits a large degree of disorder. In the sample heat-treated at $150^{\circ} \mathrm{C}$, the HRTEM exhibits features very similar to those observed in the as-deposited sample, albeit part of the former disordered region is already replaced by crystalline regions growing in from the crystalline layers on either side of the interfacial region, the thickness of which is $3.4 \mathrm{~nm}$. After 15 minutes at $245^{\circ} \mathrm{C}$, the interfacial region becomes thinner $(1.8 \mathrm{~nm})$ being gradually consumed by the advancing crystalline regions. Some amount of disorder is still present at this stage. After 15 minutes at $365^{\circ} \mathrm{C}$, the interfacial region appears to be fully crystalline.
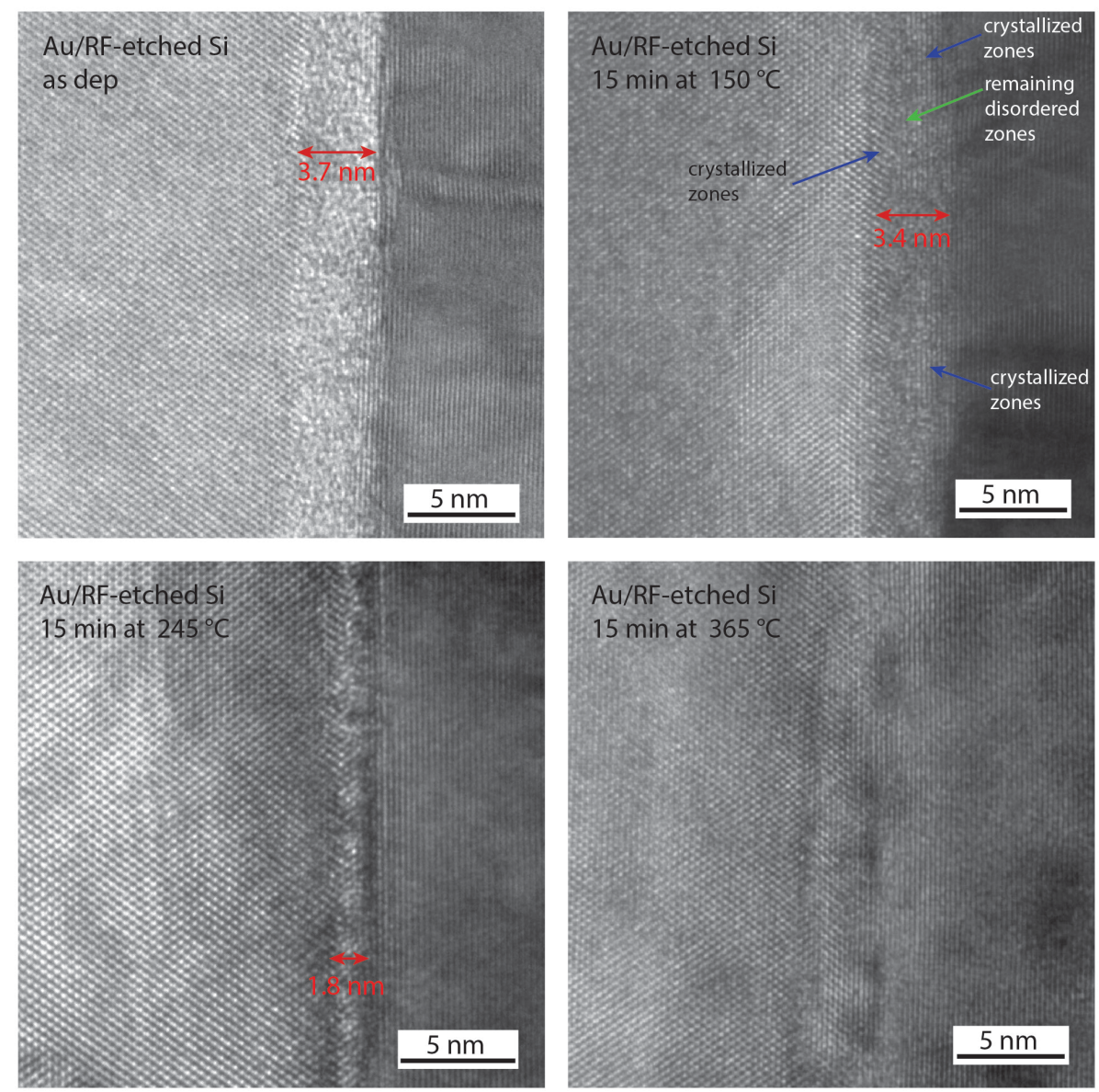

Fig. 2: HRTEM image of the interface formed for the Au/RF-etched Si sample a) in the as deposited state, b) after a 15 min heat-treatment at $150^{\circ} \mathrm{C}$ and c) after a 15 min heattreatment at $245^{\circ} \mathrm{C}$ and d) after a $15 \mathrm{~min}$ heat-treatment at $365^{\circ} \mathrm{C}$. 
Attempts were made to get deeper insight into the perturbed region chemical composition using STEM-EDX on a selection of samples. Fig. 3 shows the profile obtained for the as-deposited sample and after a heat treatment at $245^{\circ} \mathrm{C}$. Both composition profiles are virtually the same, which indicates that the heat-treatment did not induce significant interdiffusion of gold and silicon into each other, in accordance with the expectations from the equilibrium phase diagram of the Au-Si system. A somewhat more gradual transition from silicon to gold on the silicon side, however, tends to indicate that gold and silicon coexist over a certain range of thicknesses in the interfacial region. In the as-deposited sample, this coexistence most likely originates from kinetic implantation of gold ions into silicon during sputtering and can be seen as a solid-solution located in the disordered interfacial region. In the sample that was heattreated at $245^{\circ} \mathrm{C}$, the situation is somehow different. The interfacial region was shown to have partially crystallized, which was most likely made possible through a mechanism similar to the one involved in the solidification of divorced hypoeutectic alloys. In a nutshell, the gold-silicon "solid-solution" observed in the as-deposited sample evolves towards a more stable configuration, which results in a separation of the interfacial region into gold and silicon crystalline regions growing in from their respective sides. The average amount of clusters of each element varies over the thickness of the interfacial region, which gives rise to the more gradual transition from silicon to gold observed on the silicon side.

It is worth noting that reliable local values for the exact composition in the interfacial region could not be measured because of its very limited lateral extension compared to the thickness of the sample.

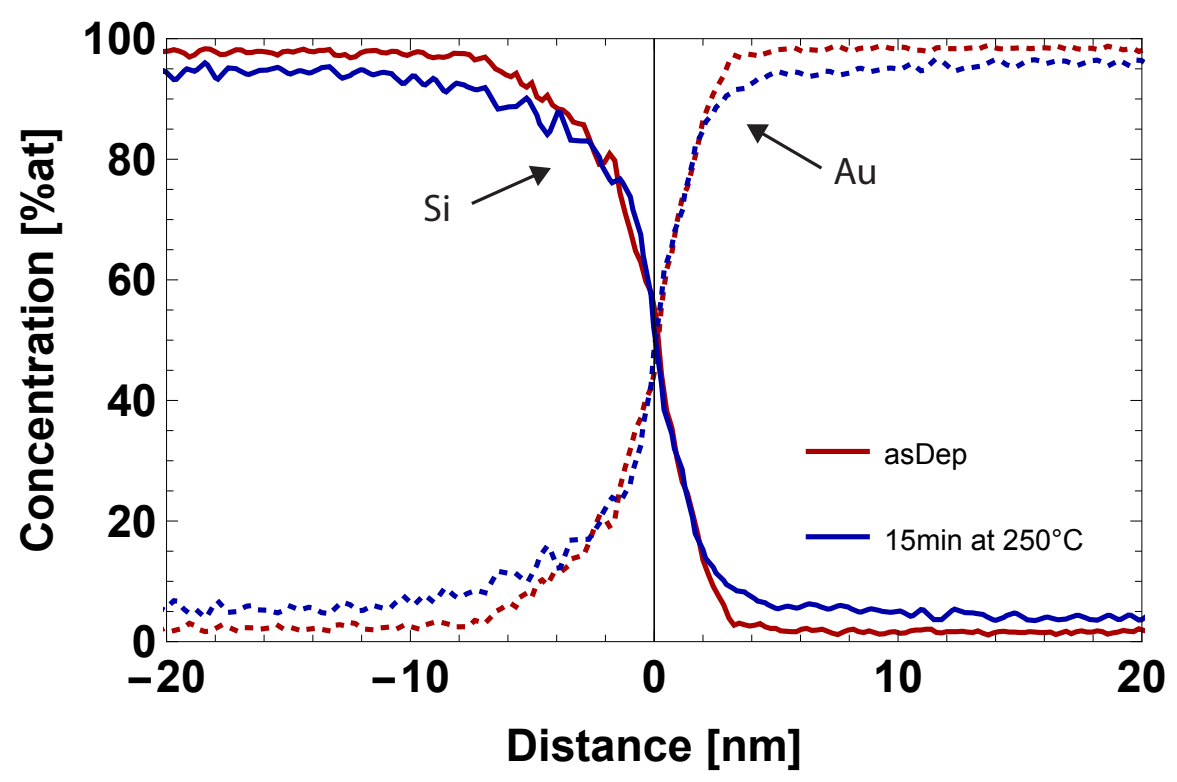

Fig. 3: STEM-EDX profile of the Au/Si interface for the as-deposited sample and after a heat-treatment at $245^{\circ} \mathrm{C}$. 


\section{ii. Ti/Si interface}

Fig. 4 shows a typical example of HR-STEM images obtained for Ti/Si samples. The Ti-Si stack is orientated such that the silicon is located on the bottom part of the image. The whole sample is oriented along the silicon (110) zone axis. The silicon clearly appears to be crystalline. The titanium similarly exhibits interference fringes, although these are less visible. It is important to recognize that the reduced quality of these fringes is most likely not an indication of a possible reduction of crystallinity. Titanium is typically expected to affect the signal quality by combining both a higher atomic number and a larger thickness because it is harder than the capping Al-layer. Its crystallographic orientation among neighbouring crystallites is furthermore expected to be random, while the sample orientation makes crystal interference fringes visible only when they are aligned along the silicon (110) zone axis.

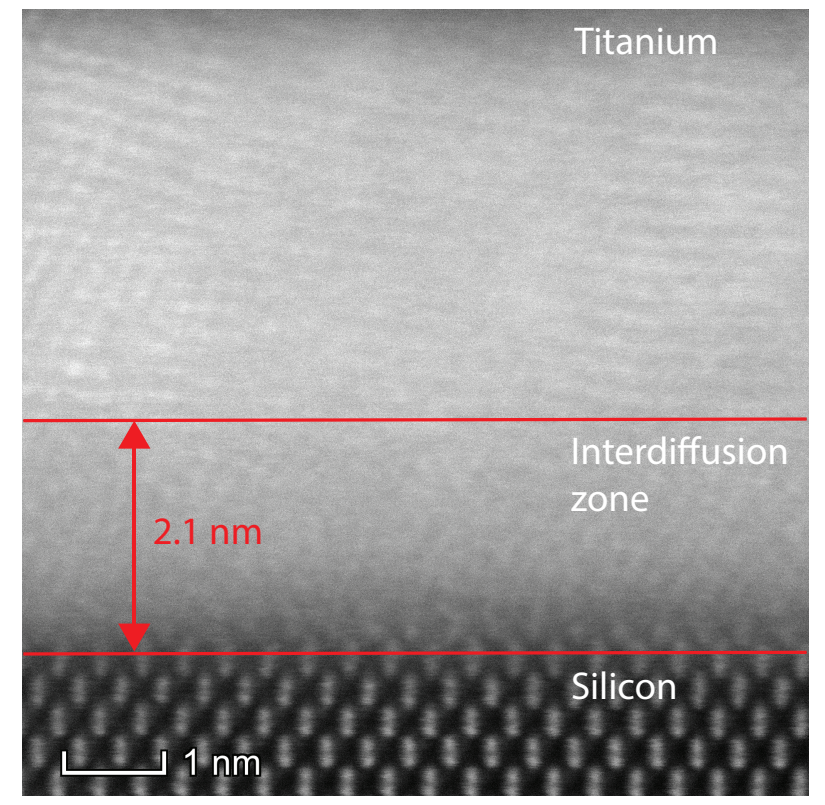

Fig. 4: HRSTEM images of the interface formed for the Ti/Si sample after a 30 min heattreatment at $350^{\circ} \mathrm{C}$.

The interface between the titanium layer and the silicon was observed to be diffuse and characterized by a region, in which no interference fringes could be observed, suggesting a high level of disorder. This so-called "disordered region" was measured to be roughly $2 \mathrm{~nm}$ thick for all the samples tested and observed to be always darker than the crystalline titanium layer, suggesting that it contains larger amounts of silicon.

The chemistry of the samples tested was further explored by STEM-EDX. Fig. 5 shows the profiles obtained for two different samples (as-deposited and heat-treated for $3 \mathrm{~h}$ at $350^{\circ} \mathrm{C}$ ) together with the corresponding HAADF intensity profiles. In Fig. 5b., it should be noted that the values on the left-hand side of the dashed red line are not given because the sample was damaged away from the interface due to preferential polishing of the metallic layers as compared to silicon, which prevented us from getting any reliable data in this region. Several interesting features were, however, observed on the right-hand side of the red dashed line. The intensity of the HAADF signal is known to be 
the largest for heavy elements. Fig. 5 consistently shows that the HAADF peak intensity is located in the region that comprises both the titanium and the disordered layers. It can be further observed that, in the as-deposited sample, the titanium is distributed uniformly across this region.
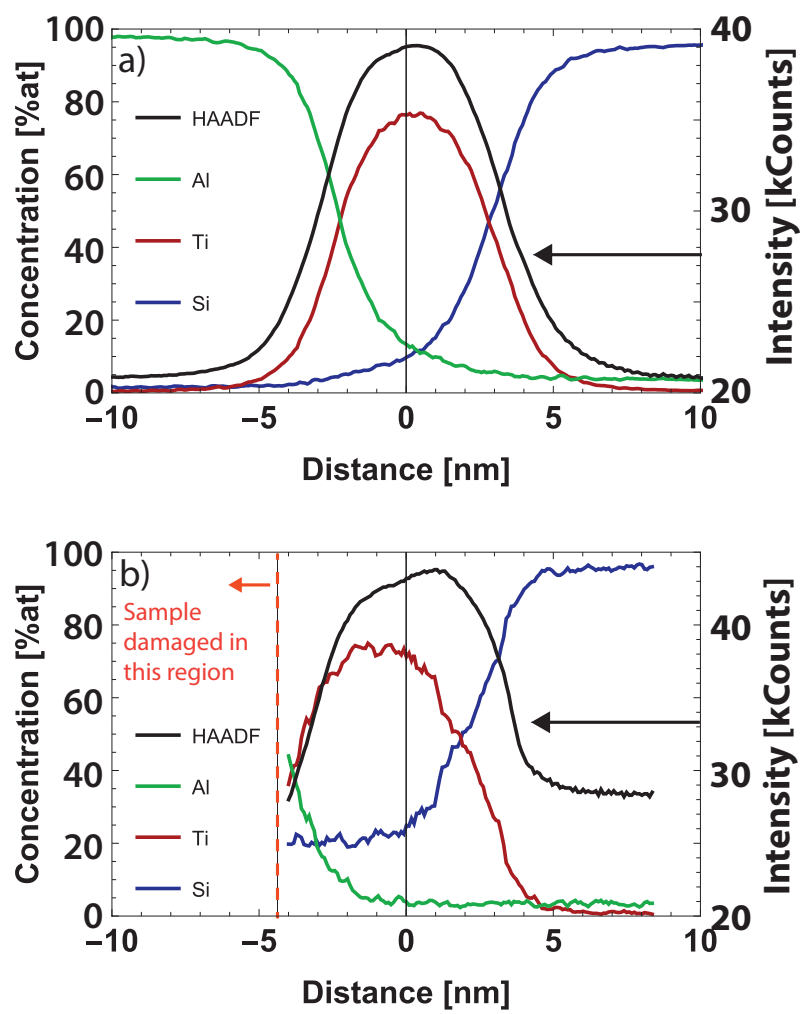

Fig. 5: STEM-EDX profile of the Ti/Si interface for a) the as-deposited sample and b) after heat-treatments performed at $350^{\circ} \mathrm{C}$ for $3 \mathrm{~h}$ together with the corresponding HAADF profiles.

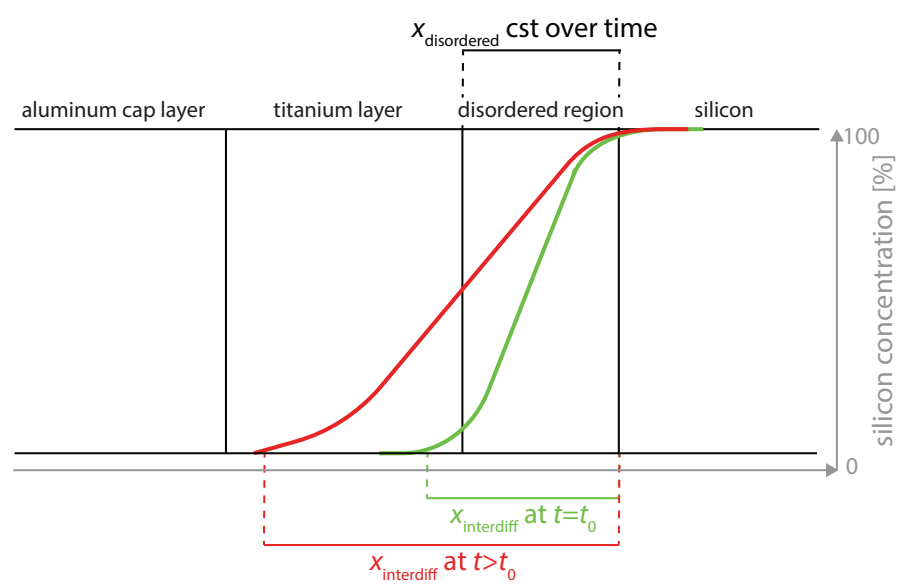

Fig. 6: Schematic of the effect of heat-treatments on the chemical composition of Ti/Si samples. 
A $3 \mathrm{~h}$ heat-treatment, however, changes this distribution with the titanium peak that is shifted towards the aluminium side due to silicon diffusion into the amorphous layer and eventually reaches the titanium layer, as depicted in Fig. 6. Interestingly enough, the thickness of the disordered region and the silicon diffusion depth consequently appear to be unrelated, although silicon diffusion most likely involves a change in the disordered region chemistry. Just as for the $\mathrm{Au} / \mathrm{Si}$ samples, the disordered regions were very thin, which prevented us from determining precisely their chemical composition.

\section{b. Thermal boundary conductance evolution with heat-treatments}

The TBC values obtained for $\mathrm{Au} / \mathrm{Si}$ are given in Fig. 7. The TBC of the as deposited sample was measured to be $124 \mathrm{MW} / \mathrm{m}^{2} \mathrm{~K}$. The $\mathrm{TBC}$ was then found to decrease for all heat-treatments performed. A value close to $90 \mathrm{MW} / \mathrm{m}^{2} \mathrm{~K}$ was measured in all the heattreated samples tested.

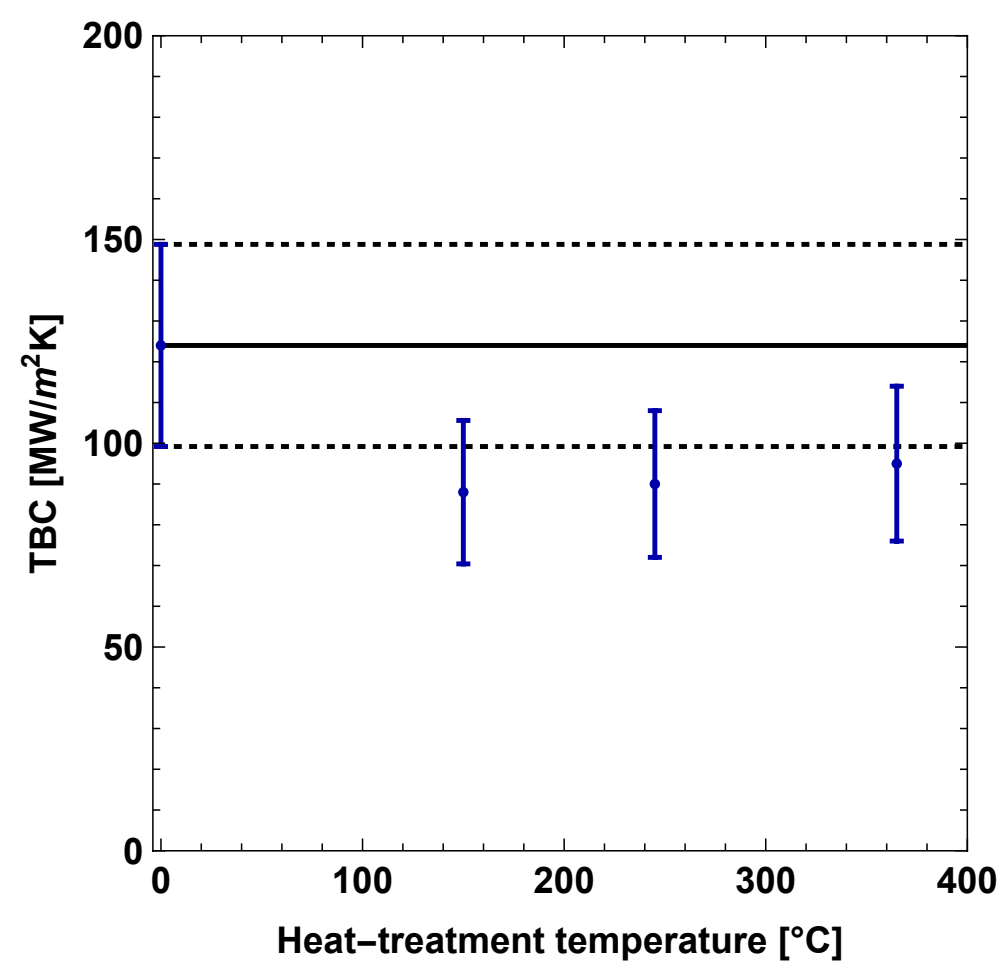

Fig. 7: TBC measured at the $\mathrm{Au} / \mathrm{Si}$ interface for the as-deposited sample and after hea treatments performed at 150,245 and $365^{\circ} \mathrm{C}$.

Fig. 8 shows the results obtained for the Ti/Si samples. The thermal boundary conductance was measured to be $272 \mathrm{MW} / \mathrm{m}^{2} \mathrm{~K}$ for the as deposited sample. It was then observed to decrease continuously with increasing heat-treatment duration. After $3 \mathrm{~h}$ at $350^{\circ} \mathrm{C}$, the $\mathrm{TBC}$ was measured to be $148 \mathrm{MW} / \mathrm{m}^{2} \mathrm{~K}$, which corresponds to a decrease of roughly 50 pct as compared to the as-deposited sample. 


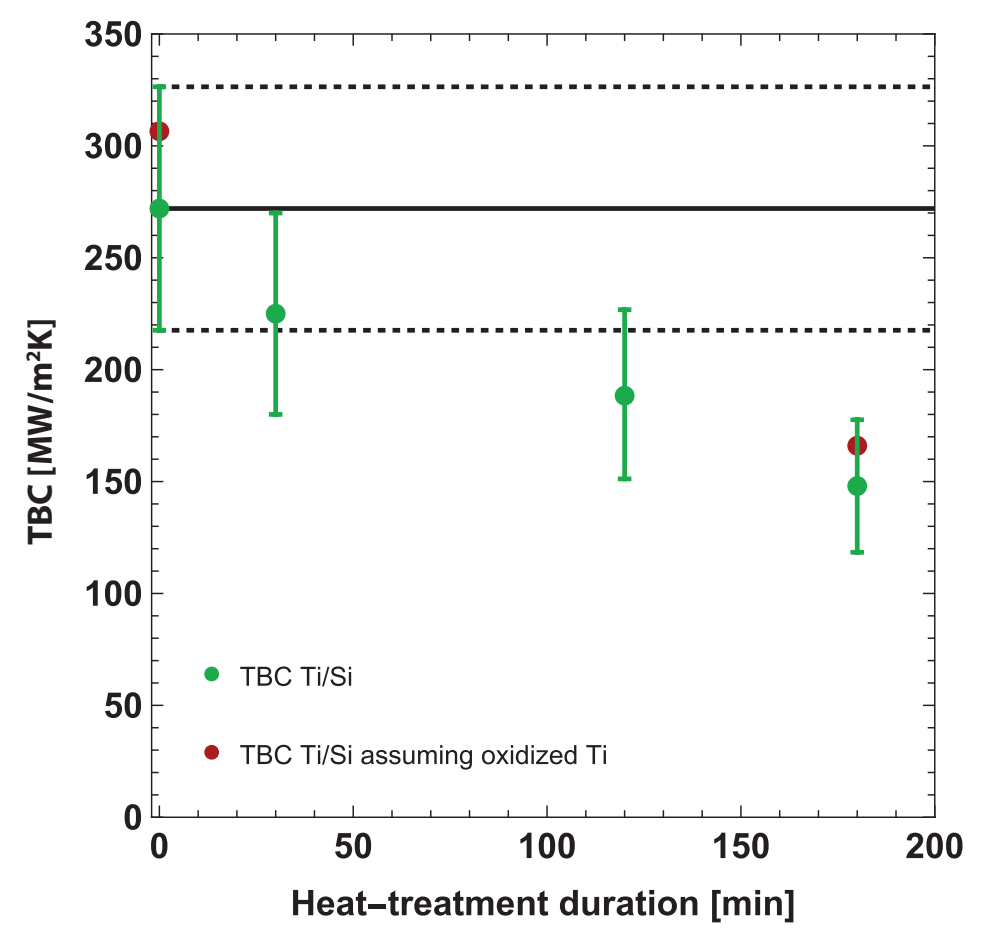

Fig. 8: TBC measured at the $\mathrm{Au} / \mathrm{Si}$ interface for the as-deposited sample and after hea treatments performed at 150,245 and $365^{\circ} \mathrm{C}$.

\section{DISCUSSION}

\section{a. TDTR measurement sensitivity to the disordered intermixed region's thermal properties}

All TBC values were extracted from TDTR measurements without knowing the exact properties of the regions where intermixing is present. A mixture of metal and silicon is most likely formed, whose thermal properties are assumed to be close to the thermal properties of crystalline silicide. Therefore: i) its thermal conductivity was set to 20 $\mathrm{W} / \mathrm{mK}(23)$ and ii) its volumetric heat capacity is assumed to be close to the one of the pure metal $(24,25)$. These rough estimates may, however, be somewhat off from reality and serve just as first approximations. Typically, the thermal conductivity is known to be much lower in highly disordered materials than in crystals. The validity of the TBC values obtained using these assumptions thus depends on the sensitivity $S$ of the TDTR measurement to the thermal properties of the regions, in which intermixing is present. In a nutshell, $S_{\mathrm{i}}$ describes to which extent the measurement will vary when introducing some uncertainty on parameter $i$. An $S_{\mathrm{i}}$ value close to zero means that even large errors on parameter $i$ will not affect the end result significantly. Inversely, errors on parameter $i$ will become increasingly critical with increasing $S_{\mathrm{i}}$ values. For both $\mathrm{Au} / \mathrm{Si}$ (14) and Ti/Si interfaces (see Fig. 9), the sensitivity of the TDTR measurement to the thermal conductivity, volumetric heat capacity and thickness of the region, in which intermixing 
is present falls close to zero, which gives us confidence that our assumptions will not affect significantly the extracted TBC value.

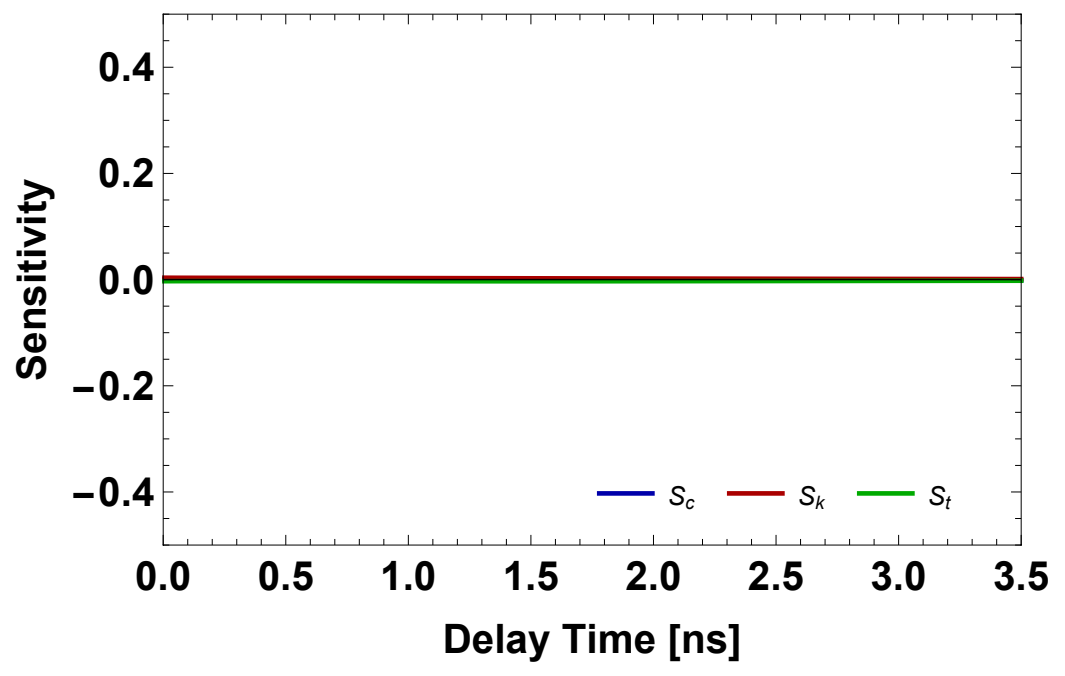

Fig. 9: Ti/Si samples TDTR measurement sensitivity to the interfacial region heat capacity, thermal conductivity and thickness.

\section{b. TBC vs. interface characteristics}

The TBC at the $\mathrm{Au} / \mathrm{Si}$ interface was found to decrease from $124 \mathrm{MW} / \mathrm{m}^{2} \mathrm{~K}$ in the as deposited state to roughly $90 \mathrm{MW} / \mathrm{m}^{2} \mathrm{~K}$ after heat treatments of $15 \mathrm{~min}$ at $150^{\circ} \mathrm{C}, 245^{\circ} \mathrm{C}$ and $365^{\circ} \mathrm{C}$. These findings were rationalized as follows: the interfacial roughness was observed to be identical and very low in all samples, suggesting that it is not likely to influence the TBC values. Possible interdiffusion is another parameter that might influence the TBC. As shown in Fig. 3, however, the heat-treatments performed were not found to promote any interdiffusion between gold and silicon, which is in line with what would be expected from the equilibrium phase diagram. It is consequently not believed to be the factor that is responsible for the TBC decrease observed in Fig. 7. The remaining parameter that is likely to influence the TBC is the structure of the interfacial region. In the as deposited sample, the interfacial region was found to consist of a highly disordered mixture of gold and silicon that can be seen as a (unstable) solid solution, which is metallic in nature. The overall thermal transport throughout this region is consequently controlled by i) a TBC at the interface between gold and the interfacial region that is controlled by electrons and ii) a TBC at the interface between the interfacial region and silicon that is controlled by phonons. The TBC resulting from this last contribution, depends on the properties of the interfacial region rather than on the properties of gold with a gradient in chemical composition that allows for a smooth transition between gold and silicon, which was theoretically shown to be possibly an efficient way to improve thermal transport across highly mismatched interfaces $(4,5)$. In samples that were heat-treated, on the other hand, the interfacial region was shown to be characterized by the separation of gold and silicon growing on their respective layers. 
The TBC is consequently controlled by the properties of gold rather than by the properties of a smoothly graded interface, thus hampering heat transfer across the interface. The different heat-treatment temperatures did not have a strong effect on the TBC, which most likely indicates that the chemical separation of gold and silicon in the interfacial region dominates over a possible effect of the atomic order.

The TBC at the Ti/Si interface was observed to decrease from $272 \mathrm{MW} / \mathrm{m}^{2} \mathrm{~K}$ for the as deposited sample down to $148 \mathrm{MW} / \mathrm{m}^{2} \mathrm{~K}$ for the samples that was heat-treated during 3h. The interfacial roughness was found to be similar and very low for all samples, suggesting that is unlikely to influence the TBC. A change in the different layers chemical composition is another parameter that may influence the TBC. A change in the layers' chemical composition due to interdiffusion may affect the TBC significantly. STEM-EDX measurements combined with HAADF profiles shown in Fig. 5 indicate that significant interdiffusion is taking place during heat-treatments. Silicon was shown to be present in the disordered region in the as-deposited sample, while it was shown to be present both in the disordered region and in the titanium layer after a $3 \mathrm{~h}$ heat-treatment at $350^{\circ} \mathrm{C}$. This inevitably leads to significant changes in the chemical composition of the interfacial region, which is believed to be responsible for the change of the TBC observed in Fig. 8. To get a deeper understanding on the effect of interdiffusion on TBC, a detailed exploration of the disordered region chemical composition would be necessary. This could not be achieved in the present work because of the thinness of this region.

Alternatively, Ti oxidation might affect the TBC, especially if the degree of oxidation varies from one sample to another. The temperature drop within an oxidized layer is expected to be larger than the temperature drop in the corresponding pure metal layer, which has a larger thermal conductivity and might artificially lower the TBC value. Although much care was taken to avoid titanium oxidation (in-situ heat-treatments, storage under vacuum and measurements performed rapidly after deposition), the TBC at $\mathrm{Ti} / \mathrm{Si}$ interface assuming that $\mathrm{Ti}$ was transformed into $\mathrm{TiO}_{2}$ was evaluated for the asdeposited samples and for the sample heat-treated at $350^{\circ} \mathrm{C}$ during $3 \mathrm{~h}$. The thermal conductivity and thermal capacity of $\mathrm{TiO}_{2}$ were set to $5 \mathrm{~W} / \mathrm{mK}(26,27)$ and $2.96 \mathrm{~J} / \mathrm{m}^{3} \mathrm{~K}$, respectively (28). Under such a configuration, the TBC was found to increase slightly in both cases (red points in Fig. 8) but the observed difference is unlikely to explain the general trend observed in Fig. 8.

\section{CONCLUSION}

This work aimed at experimentally exploring a possible influence of interdiffusion and/or interfacial disorder on the thermal boundary conductance of metal/dielectric interfaces. Therefore, $\mathrm{Au} / \mathrm{Si}$ and $\mathrm{Ti} / \mathrm{Si}$ samples that underwent different heat treatments were studied. The results obtained indicate that i) for interfaces with significant phonon DOS mismatch higher TBC values can be achieved in presence of a smoothly graded interface, as compared to a chemically abrupt interface, while the atomic arrangement was not observed to have any significant effect and ii) increasing interdiffusion decreases the TBC at interfaces that would in their clean state have a high TBC, as 
observed in the Ti/Si samples. For the latter, a potential contribution of oxidation of the Ti layer could not be excluded but was shown to have only limited effect on the overall TBC dependence on the heat-treatment duration.

\section{SUPPLEMENTAL MATERIAL}

The supplemental material provides information about temperature cycle that were used for the $\mathrm{Au} / \mathrm{Si}$ samples heat-treatment and gives each sample characteristics (heattreatment parameters, thickness of the layers and TBC). An explanation about the location at which the interface is placed is also provided.

\section{ACKNOWLEDGEMENTS}

The authors are grateful acknowledge the SNSF (Project No 200021_149290) for its financial support and Prof. O. Martin from the Nanophotonics and Metrology Laboratory (NAM, EPFL) for providing the laser source. Dr. A. Magrez from the iPhys platform (EPFL) warmly thanked for his support with the Empyrean diffractometer, Dr. C. Monachon for having provided the code that enables TDTR data analysis and the Interdisciplinary Centre for Electronic Microsocpy (CIME, EPFL) for its support with TEM samples preparation and imaging.

\section{BIBLIOGRAPHY}

1. Lyeo, H. K., \& Cahill, D. G., Thermal conductance of interfaces between highly dissimilar materials. Physical Review B, 73(14) (2006) 144301, DOI: 10.1103/PhysRevB.73.144301

2. Twu, C. J., \& Ho, J. R., Molecular-dynamics study of energy flow and the Kapitza conductance across an interface with imperfection formed by two dielectric thin films. Physical Review B, 67(20) (2003) 205422, DOI: 10.1103/PhysRevB.67.205422

3. Beechem, T., Graham, S., Hopkins, P., \& Norris, P., Role of interface disorder on thermal boundary conductance using a virtual crystal approach. Applied Physics Letters, 90(5) (2007), 054104, DOI: $10.1063 / 1.2437685$.

4. Stevens, R. J., Zhigilei, L. V., \& Norris, P. M., Effects of temperature and disorder on thermal boundary conductance at solid-solid interfaces: Nonequilibrium molecular dynamics simulations. International Journal of Heat and Mass Transfer, 50(19-20) (2007) 3977-3989, DOI: 10.1016/j.ijheatmasstransfer.2007.01.040

5. Liang, X. G., \& Sun, L., Interface structure influence on thermal resistance across double-layered nanofilms. Microscale Thermophysical Engineering, 9(3) (2005) 295-304, DOI: $10.1080 / 10893950500196469$

6. Beechem, T., \& Hopkins, P. E., Predictions of thermal boundary conductance for systems of disordered solids and interfaces. Journal of Applied Physics, 106(12) (2009), 124301, DOI: 10.1063/1.3267496.

7. Giri, A., Braun, J. L., \& Hopkins, P. E., Effect of crystalline/amorphous interfaces on thermal transport across confined thin films and superlattices. Journal of Applied Physics, 119(23) (2016) 235305, DOI: 10.1063/1.4953683

8. Giri, A., Hopkins, P. E., Wessel, J. G., \& Duda, J. C., Kapitza resistance and the thermal conductivity of amorphous superlattices. Journal of Applied Physics, 118(16) (2015) 165303, DOI: 10.1063/1.4934511

9. Li, M., Zhang, J., Hu, X., \& Yue, Y., Thermal transport across graphene/SiC interface: effects of atomic bond and crystallinity of substrate. Applied Physics A, 119(2) (2015) 415-424, DOI: 10.1007/s00339-0159066-7 
10. Gordiz, K., \& Henry, A., Phonon transport at interfaces between different phases of silicon and germanium. Journal of Applied Physics, 121(2) (2017) 025102, DOI: 10.1063/1.4973573

11. Hopkins, P. E., Norris, P. M., Stevens, R. J., Beechem, T. E., \& Graham, S., Influence of interfacial mixing on thermal boundary conductance across a chromium/silicon interface. Journal of Heat Transfer, 130(6) (2008) 062402, DOI: 10.1115/1.2897344

12. Lur, W., \& Chen, L. J., Growth kinetics of amorphous interlayer formed by interdiffusion of polycrystalline Ti thin - film and single - crystal silicon. Applied physics letters, 54(13) (1989) 1217-1219, DOI: $10.1063 / 1.100720$

13. Chen, L. J., Solid state amorphization in metal/Si systems. Materials Science and Engineering: R: Reports, 29(5) (2000) 115-152, DOI: 10.1016/S0927-796X(00)00023-1

14. Blank, M., \& Weber, L. (2019). Towards a coherent database of thermal boundary conductance at metal/dielectric interfaces. Journal of Applied Physics, 125(9), 095302, DOI: 10.1063/1.5085176

15. Hull, R. (Ed.). (1999). Properties of crystalline silicon (No. 20). IET, ISBN: 0852969333

16. Lin, Z., Zhigilei, L. V., \& Celli, V., Electron-phonon coupling and electron heat capacity of metals under conditions of strong electron-phonon nonequilibrium. Physical Review B, 77(7) (2008) 075133, DOI: 10.1103/PhysRevB.77.075133

17. Blank, M., \& Weber, L., Influence of the thickness of a nanometric copper interlayer on Au/dielectric thermal boundary conductance. Journal of Applied Physics, 124(10) (2008) 105304, DOI: $10.1063 / 1.5030049$

18. Björck, M., \& Andersson, G., GenX: an extensible X-ray reflectivity refinement program utilizing differential evolution. Journal of Applied Crystallography, 40(6) (2007) 1174, DOI: $10.1107 / \mathrm{S} 0021889807045086$

19. Kang, K., Koh, Y. K., Chiritescu, C., Zheng, X., \& Cahill, D. G., Two-tint pump-probe measurements using a femtosecond laser oscillator and sharp-edged optical filters. Review of Scientific Instruments, 79(11) (2008) 114901, DOI: 10.1063/1.3020759

20. Monachon, C., Thermal boundary conductance between metals and dielectrics, PhD Thesis, (2013) DOI: 10.5075/epfl-thesis-5872

21. Cahill, D. G., Analysis of heat flow in layered structures for time-domain thermoreflectance. Review of scientific instruments, 75(12) (2004) 5119, DOI: 10.1063/1.1819431

22. Haynes, W. M. (Ed.), CRC handbook of chemistry and physics. CRC press, (2014).

23. Neshpor V.S., The thermal conductivity of the silicides of transition metals, Journal of engineering physics, 15 (1968) 750, DOI: 10.1007/BF00829703

24. Hamaoui G., Horny N., Hua Z., Zhu T., Robillard J-F., Fleming A., Ban H., Chirtoc M., Electronic contribution in heat transfer at metal-semiconductor and metal silicide-semiconductor interfaces, Scientific Reports, 8 (2018) 11352, DOI: 10.1038/s41598-018-29505-4.

25. Ye N., Feser J.P., et al., Sadasivam S., Fisher T. S., Wang T., Ni C., Janotti A., Themal transport across metal silicide-silicon interfaces: An experimental comparison between epitaxial and nonepitaxial interfaces, Physical Review B, 95 (2017) 85430, DOI: 10.1103/PhysRevB.95.085430

26. Lee, S. M., Cahill, D. G., \& Allen, T. H., Thermal conductivity of sputtered oxide films. Physical Review B, 52 (1) (1995) 253, DOI: 10.1103/PhysRevB.52.253.

27. Thurber, W. R., \& Mante, A. J. H., Thermal Conductivity and Thermoelectric Power of Rutile (Ti O 2). Physical Review, 139(5A) (1965) A1655, DOI : 10.1103/PhysRev.139.A1655

28. McDonald, H. J., \& Seltz, H., The heat capacities of titanium dioxide from 68-298 K. The thermodynamic properties of titanium dioxide. Journal of the American Chemical Society, 61(9) (1939) 2405-2407, DOI : $10.1021 / \mathrm{ja} 01878 \mathrm{a} 040$. 